Nouvelle plateforme de connaissances sur les protéines humaine

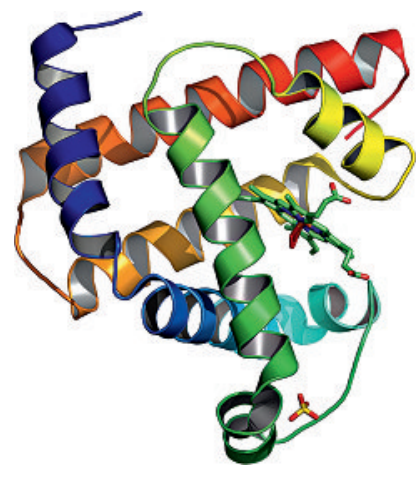

Le SIB, Institut Suisse de Bioinformatique, et GeneBio ont annoncé le lancement de neXtProt, une plateforme de connaissances sur les protéines humaines. Cette ressource innovante offre aux chercheurs en sciences de la vie un large éventail d'informations sur toutes les protéines humaines par le biais d'une interface intuitive. Avec plus de 51000 visiteurs depuis la première version Beta lancée en janvier de cette année, neXtProt promet de devenir une ressource incontournable pour tout chercheur en sciences de la vie en quête d'informations de qualité sur les protéines humaines.

\section{Neu: «kids+media»}

Das Institut für Populäre Kulturen der Universität Zürich und das Schweizerische Institut für Kinderund Jugendmedien haben eine neue Zeitschrift lanciert. «kids+ media» ist kostenlos und erscheint online auf www.kids-media.uzh.ch. Thema der ersten Nummer ist «Vorzeit, Frühzeit, Vergangenheit». «Zur Grundidee von kkids+media> gehört, dass wir neben arrivierten Wissenschaftlerinnen und Wissenschaftlern auch dem Nachwuchs eine Gelegenheit zum Publizieren geben wollen», sagt Frau Tomkowiak, die $\mathrm{zu}$ den Herausgeberinnen gehört. Die Texte sollen sich vor allem an ein wissenschaftliches Publikum richten, dabei aber allgemeinverständlich geschrieben sein.

(Universität Zürich)

\title{
Lagerfläche für «Velos für Afrika» gesucht
}

Die Recycling-Werkstätten Gump- \& Drahtesel suchen im Grossraum Basel/Liestal oder in anderen zentral gelegenen Regionen der Schweiz für das Projekt «Velos für Afrika» kostengünstige oder kostenlose Flächen zum Lagern von gespendeten Velos. Interessierte kontaktieren bitte Matthias Maurer, matthias.maurer@gump-drahtesel.ch. Die Velos des Projekts sind in Afrika gefragter denn je. Aktuell ist auch der Bau von Dreirädern

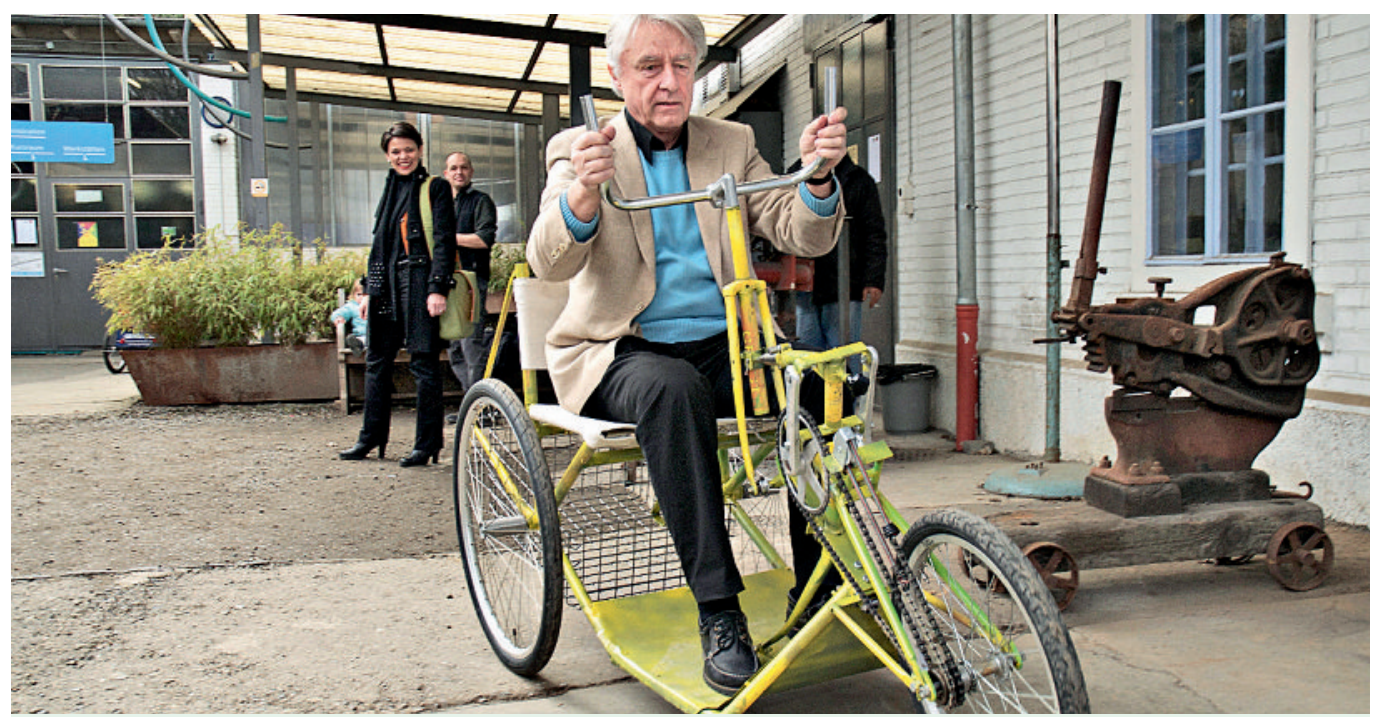

Auch das Fahren auf einem Dreirad will gelernt sein: Emil auf einem Velo für Gehbehinderte in Burkina Faso.

\section{Neuer Beruf: Assistent/in Gesundheit und Soziales}

Die nationale Dachorganisation der Arbeitswelt Gesundheit OdASanté und die nationale Dachorganisation der Arbeitswelt Soziales SAVOIRSOCIAL haben einen neuen Beruf geschaffen: Die zweijährige Grundbildung zur Assistentin Gesundheit und Soziales mit eidgenössischem Berufsattest soll praktisch begabten Personen die Möglichkeit bieten, im Gesundheits- und Sozialbereich Fuss zu fassen. Die neue Ausbildung bie-

\section{Journée nationale Aide et soins à domicile 2011}

«Vous soignez. Avec notre soutien. Aide et soins à domicile.» Tel est le slogan de la Journée nationale Aide et soins à domicile qui se deroulera le 3 septembre 2011. Les organisations d'aide et de soins à domicile vont présenter leurs prestations au grand public. Le slogan souligne la grande importance des proches soignants; il met l'accent sur un groupe de personnes dont l'engagement n'est pas encore vraiment perçu par la société. Le slogan met également en évidence l'utilité de l'aide et des soins à domicile pour les personnes concernées. Les services d'aide et de soins à domicile épaulent les proches grâce à des offres de soutien et ils les aident là où c'est nécessaire. Des informations sur les manifestations locales peuvent être obtenues auprès des associations cantonales. tet Jugendlichen nach der obligatorischen Schulbildung einen Einstieg in Betreuungs- und Pflegeaufgaben im Gesundheits- und Sozialwesen. Diese Grundbildung steht auch erwachsenen Personen offen, die einen Einstieg ins Berufsfeld Gesundheit und Betreuung suchen, oder aber eine mehrjährige Praxiserfahrung durch Nachholbildung formell anerkennen lassen wollen.

(OdASanté)

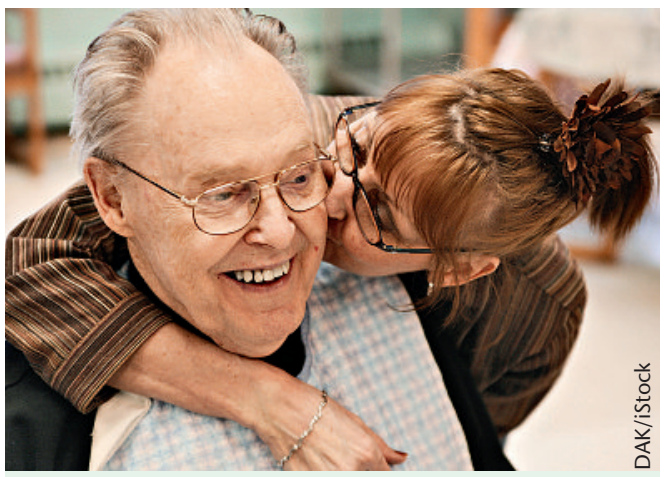

L'engagement et la grande importance des proches soignants n'est pas encore vraiment perçu par la société. 